\title{
Tracking of spatial information in narratives
}

\author{
WILLIAM H. LEVINE and CELIA M. KLIN \\ State University of New York, Binghamton, New York
}

\begin{abstract}
Three experiments were conducted to investigate the process by which location information in narratives is represented in memory and the nature of the resulting memory representation. In Experiments 1 and 2, the results of a recognition task demonstrated that location shifts led to an immediate decrease in the accessibility in memory of protagonists' former locations. In Experiment 3, regardless of the amount of backgrounding after the last mention of the critical location ("the forest"), reference to an implied, location-typical entity ("the trees") was read equally fast as long as the protagonist remained in that location. In contrast to previous findings, we conclude that when location information is salient in a narrative it is included in readers' situation models, being updated immediately and remaining highly accessible even several sentences after it was last mentioned.
\end{abstract}

The purpose of a narrative is to tell a story or describe a set of events. In accord with this, many theorists (e.g., Bransford, Barclay, \& Franks, 1972; Garrod \& Sanford, 1990; Glenberg, Meyer, \& Lindem, 1987; Johnson-Laird, 1983; Morrow, Greenspan, \& Bower, 1987; van Dijk \& Kintsch, 1983;Zwaan \& Radvansky, 1998) have proposed that motivated readers are primarily concerned with constructing a situation model, a mental representation of the world described by the narrative. Although the concept of a situation model is incompletely specified, the spatial component of situation models has been extensively investigated, with numerous studies asking whether location information is included in a situation model. That is, does spatial information retain a privileged status in memory, being held in implicit focus (Garrod \& Sanford, 1990) or long-term working memory (Ericsson \& Kintsch, 1995; Zwaan \& Radvansky, 1998)? The importance of understanding how readers represent spatial information is due to the central role that location often plays in narratives. For example, knowledge of a character's location aids in the comprehension of "situational anaphors" (Garrod \& Sanford, 1982) - the types of entities, objects, and actions that may be referred to without their having been introduced explicitly, such as a menu in a restaurant (see, e.g., Schank \& Abelson, 1977).

Portions of these data were reported at the 39th Annual Meeting of the Psychonomic Society in Dallas, November 1998. We thank Cynthia Connine and David Payne for helpful comments on this research. Joseph Magliano and G. A. Radvansky provided insightful comments on an earlier version of this paper, as did Alexandria Guzmán, who also translated materials that were generously provided by Manuel de Vega. Special thanks go to Sarah Burt and Kristin Weingartner for their help in all phases of Experiments 1 and 2 and for their comments on the paper. Finally, we thank Rachel Altschuler, Stacey Greenblatt, Lia Kieren, Tara Jans, Dawn Melzer, Oscar Molina, and Emily Scauri for their assistance with data collection. Correspondence concerning this article should be addressed to W. H. Levine, who is now at the Department of Psychology, University of North Carolina, Chapel Hill, NC 27599-3270 (e-mail: whlevine@email.unc.edu).
Despite the extensive investigation of the question "Do readers represent spatial information in their situation models?" a definitive answer has not emerged. For instance, although a number of studies have suggested that readers are able to represent an extensive amount of spatial detail in their situation models (e.g., Haenggi, Kintsch, \& Gernsbacher, 1995; Morrow et al., 1987), in these studies researchers have employed tasks that strongly encouraged participants to pay special attention to spatial detail, raising the possibility that such findings do not generalize to more naturalistic reading. Consistent with this, Zwaan and van Oostendorp (1993) found that when readers were given instructions to pay close attention to spatial details, they read spatial information more slowly, suggesting that they were doing extra processing that would not have occurred in a natural reading situation. Moreover, using a regression analysis, Zwaan, Radvansky, Hilliard, and Curiel (1998) found that unless readers memorized a map of the setting of a narrative prior to reading, spatial discontinuities (i.e., changes in location) did not contribute to increased reading time, whereas other situational discontinuities, such as time shifts, did. Finally, Hakala (1999) found that readers did not make location-updating inferences while reading naturalistic texts unless they were instructed to pay careful attention to spatial details.

In contrast, O'Brien and Albrecht (1992; see also Black, Turner, \& Bower, 1979) found that at least some spatial information is encoded even without special instructions. They found that readers experienced comprehension difficulty when they encountered information (e.g., "She decided to go outside the health club") that was inconsistent with setting information (e.g., "Kim stood outside the health club") presented several sentences earlier. Thus, under relatively natural reading conditions, readers sometimes encode at least basic location information into their situation models.

De Vega (1995) proposed two process models that reflect the nature of the debate. These models differ with regard to when readers "update," or encode, a character's 
location, and the subsequent availability of location information in their situation models. According to the onlineupdating hypothesis, readers update location information immediately upon encountering a change in location. From this point of view, in O'Brien and Albrecht's (1992) study, as soon as readers encountered the description of Kim's location they encoded this information into their situation models. It was then readily available in memory when they encountered the sentence describing her movement out of the health club. In contrast, according to the backward-updating hypothesis, updating of location information is accomplished only when knowing a character's location is necessary to maintain the coherence of a text. From this point of view, readers did not initially encode Kim's location into their situation models, and thus this information had to be reinstated through a resourceconsuming memory search when they encountered the target line (e.g., "She decided to go outside..."); that is, only when Kim's location became pertinent did readers reactivate her location from long-term memory.

To contrast these two hypotheses, de Vega (1995) had participants read short narratives such as the following:

Introduction and layout: Maria was visiting an Italian town for the first time. In one of the squares there is a bronze sculpture of a soldier riding on a horse. On one side of the square there is an old palace. Inside the palace there is a luxurious marble staircase.

Motion sentence: Maria went into the palace from the square ... or Maria went out from the palace to the square...

Conclusion: . . . and walked a few steps. She looked around with admiration.

At the end of the passage, participants made a recognition judgment to the word palace. There was no facilitation when the probe represented the protagonists' current location relative to their former location, providing no support for the online-updating hypothesis. In another experiment, however, readers experienced comprehension difficulty if reference was made to an entity (e.g., the sculpture) that was not in the current setting of the narrative. On the basis of these findings, de Vega concluded that location information is not updated immediately. Instead, updating occurs only when location information is needed for comprehension through a resource-consuming "cue-based inference process" (p. 381).

Despite de Vega's (1995) conclusions, in the present paper we ask whether there are any conditions under which a character's location is encoded and maintained in a situation model. If we assume that a limited amount of information can be included in a situation model, the choice of what to include should be influenced by a host of text characteristics such as the importance of information to the narrative. That is, we assume that readers are flexible and efficient with regard to what information is included in their situation model. De Vega's passages were short and relatively absent of any type of goals or action, and location changes occurred across short dis- tances. Thus, characters' movements may not have needed to be fully processed for the passages to be coherent. In contrast, in a narrative in which location is central to the action in the story, unique, or highly elaborated, it may be encoded and maintained in the situation model; consider, for example, a narrative in which a character is walking through a haunted house. Thus, perhaps the most fruitful question to ask is not whether readers include location information in their situation models, but under what conditions readers include location information in their situation models.

In the present set of experiments, we attempted to identify a set of text conditions under which readers immediately update their situation models to reflect location changes, without special task demands or instructions. In Experiment 1, we attempted to make location information highly salient by (1) elaborating on the initial description of the narrative locations, and (2) making the locations relevant to the characters' goals. Both of these factors should increase the probability that location information is initially encoded and should increase the probability that it remains active in memory. As in de Vega's (1995) study, the accessibility in memory of characters' current location, relative to their old location, was measured with a recognition probe presented shortly after a location shift. After finding support for the online-updating hypothesis, in Experiment 2 we began to explore the generality of this effect, examining the influence of elaborating the location information. Finally, in Experiment 3, we tested an extension of the online-updating hypothesis, asking whether location information retains a privileged status in memory even several sentences after it has been described.

\section{EXPERIMENT 1}

In Experiment 1, we asked whether there are any text conditions under which readers immediately update a change in a protagonist's location, in the absence of text that demands access to this location information, and in the absence of task demands that induce readers to track location. We used a manipulation and materials similar to those used by de Vega (1995), but several changes were made to the materials to increase the salience of the location. A sample passage can be found in Table 1. First, location information was described elaborately; in the sample passage there are several lines describing a bar and a classroom. The added elaboration should both increase the probability that location information is considered important and lead to a more interconnected network of information about the location, making it more accessible in memory (e.g., Kintsch, 1988). Second, to ensure that careful reading of the motion sentences was important for comprehension, each protagonist's motion was integrated into the story by giving the protagonist a goal or activity in the destination location; in the sample passage, the protagonist is a medical student who has plans to go out with friends to a bar. Third, de Vega's shift 
Table 1

Sample Passages From Experiments 1 and 2

\section{Introduction and Location Description}

Experiment 1

Paula was a third-year student at Cornell Medical School. Today she had an anatomy exam. She had to identify body parts. After the exam she was meeting some friends at their favorite bar, The Connection. The bar was really a home away from home. It was a bit dark and dirty, but the staff were all friendly and they served really good appetizers, including free popcorn and the hottest Buffalo wings that Paula had ever tried.

\section{Placement Sentences}

Paula walked into the classroom to start the exam. It was an odd sight with the corpses laid out on the tables.

\section{Shift Sentences}

No-shift condition: Concentrating, she got to work and started writing quickly. Time shift condition: After two hours, she was still working and writing answers. Time and location shift condition: After two hours, she went to the bar and met her friends.

\section{Postshift Sentence}

What a long day.

\section{Recognition Probe}

CLASSROOM

$$
\text { Experiment } 2
$$

\section{Introduction and Location Description}

Paula was a third-year student at Cornell Medical School. Today she had an anatomy exam. She had to identify body parts. After the exam she was meeting some friends at a bar.

\section{Placement Sentence}

Paula walked into the classroom to start the exam.

\section{Shift Sentences}

Time shift condition: After two hours, she was still working and writing answers. Time and location shift condition: After two hours, she went to the bar and met her friends.

\section{Postshift Sentence}

What a long day.

\section{Recognition Probe}

CLASSROOM

sentences mentioned both the new and the old locations, possibly suggesting to readers that the old location was still important. In the present experiment, the old location was not referred to in the location shift sentences.

Three versions of the experimental passages were used. A recognition probe, which represented the character's original location, was presented at the end of each passage. Although all three versions ended with the same sentence, they had different penultimate sentences. The first control condition was a no-shift version, with no time or location shift in the penultimate, "shift" sentence. Thus, the character's location should have been easily accessible in memory. The second control condition was a time shift version, in which there was no location shift but there was a time shift, described either as an explicit time shift (e.g., "After two hours ...") or as a completed activity (e.g., "After finishing...."). A time shift condition was included because location shifts are naturally confounded with time shifts in narratives focusing on one character (e.g., a person cannot go from a classroom to a bar without time passing). Furthermore, time shifts have been shown to reduce the accessibility of concepts occurring before them (e.g., Carreiras, Carriedo, Alonso, \& Fernández, 1997; Zwaan, 1996). Thus, to provide the appropriate control, we needed to separate the influence of location shifts from the influence of time shifts. If time shifts decrease the accessibility of concepts mentioned prior to them, then recognition latencies should be longer in the time shift condition than in the no-shift condition.

Finally, the critical condition was the time and location shift condition. It contained the same time shift as did the time shift condition, but it also included an explicit location shift (e.g., “. . . she went to the bar ...”). According to the online-updating hypothesis, readers should immediately update their representations, leading to a reduction in accessibility of the character's former location (e.g., classroom). Given this, recognition times should be longer in the time and location shift condition, where the protagonist has left the classroom, than in the time shift condition, where she remains in the classroom, despite the fact that the distance between the probe word and the last mention of the concept is equated across versions. In con- 
trast, if location information is updated only when it is needed to maintain the coherence of the narrative, there should be no difference in the recognition latencies in the time shift and time and location shift conditions, replicating de Vega's (1995) results.

\section{Method}

Participants. Seventy-five members of the SUNY Binghamton community participated in exchange for course credit or $\$ 5$. The data from 7 participants with too many outliers were replaced. ${ }^{1}$ Therefore, the analyses were based on data from 68 participants.

Materials. Twenty-one experimental passages were used, with three versions of each. The passages were all short narratives (mean length $=9.6$ sentences, range $=6-14$ sentences) similar to the example in Table 1. In all three conditions, passages began with a description of both a character and a goal, as well as an elaborate description of the destination location (e.g., the bar) where the goal was to be achieved. This was followed by one or two placement sentences, in which the character was placed in an "origin" location (e.g., a classroom) and described as being involved in an activity there (e.g., taking an exam). The penultimate, shift sentence differed across the three conditions. In the no-shift condition, the activity described in the placement sentence continued, without any explicit passage of time or a change in location. In the time shift condition, the sentence described a time shift. In the time and location shift condition, the shift sentence described the same time shift as did the time shift condition, as well as the character moving to the destination location. Across conditions, the shift sentences were equated for length and had similar syntactic structures. All three conditions ended with the same postshift sentence, which made no reference to location. After the postshift sentence, a recognition probe was presented. The probe represented the origin location from the placement sentence- that is, the current location in the no-shift and time shift conditions, and the former location in the time and location shift condition.

In addition to the experimental passages, there were 25 filler passages. All filler probes were common nouns, 7 requiring a "yes" and 18 requiring a "no" response. Across all passages, 28 probes required "yes" responses and 18 required "no" responses.

Design. The experimental passages were randomly assigned with two constraints: (1) Each participant saw one third of the passages in each of the three conditions, and (2) each version of the passage was presented to one third of the participants. Filler passages were interspersed among the experimental passages.

Procedure. The passages were presented on a video monitor. Each trial began with the words READY FOR NEXT STORY in the center of the screen. The passages were displayed one line at a time in upper- and lowercase letters. Pressing an advance key on a threebutton response box erased the current line from the screen and replaced it with the next line. At the end of the passage, the last line of text was replaced by a warning signal (XXX) centered on the screen for $500 \mathrm{msec}$. This warning signal was then replaced by the probe word, in all capital letters. The participants were instructed to decide, as quickly and accurately as possible, whether the probe was in the preceding text by pressing a yes or no key on the response box. The participants received feedback on the speed but not the accuracy of their response. Then another warning signal (???) was presented for $500 \mathrm{msec}$, followed by a two-alternative forced choice comprehension question. The participants were instructed to answer the question as accurately as possible and were given feedback on the accuracy of their response. Before reading the first experimental passage, the participants read three practice passages. To encourage careful reading, for five of the filler passages, instead of answering comprehension questions, the participants were instructed to write short continuations (i.e., about two sentences long). They were informed ahead of time that they would have to write continuations, but they did not know for which passages.

\section{Results and Discussion}

For each participant, recognition latencies over 2,000 msec and outlying latencies, as defined by Tukey's (1977) criterion, were discarded; $5.0 \%$ of the response times were dropped. ${ }^{2}$ In addition, owing to an error, the data from one of the passages were discarded. In all analyses to be reported, an alpha level of .05 was used. $t_{1}$ represents the result of analyses in which the error term was based on participant variability, and $t_{2}$ represents the result of analyses in which the error term was based on item variability. The results are displayed in Table 2 .

Recognition latencies. Recognition latencies were longer in the time and location shift condition $(954 \mathrm{msec})$ than in either the no-shift condition $(920 \mathrm{msec})\left[t_{1}(67)=\right.$ $1.99, S E M=17.11, p=.05 ; t_{2}(19)=1.92, S E M=21.91$, $p=.07]$ or the time shift condition $(895 \mathrm{msec})\left[t_{1}(67)=\right.$ $3.21, S E M=18.36, p=.002 ; t_{2}(19)=2.64, S E M=21.62$, $p=.02]$. Thus, when the participants read the shift sentence in the time and location shift condition, they deactivated or suppressed (Gernsbacher, 1990) the old location, reducing its accessibility in memory. However, because there was a change in the dimension of time as well as in the dimension of space in the time and location shift condition, the reduction in the accessibility of protagonists' former location may have been due to the location shift, the time shift, or a combination of the two. If recognition latencies for the time shift condition are compared with those for the no-shift condition, however, there is no indication that a time shift contributed to the reduction in the accessibility of the protagonist's location. In fact, recognition times were nonsignificantly shorter in the time shift condition than the no-shift condition (both $p$ s $>$.12). This suggests that time shifts only lead to the reduction in accessibility of temporally bound information, such as scenario-dependent characters or brief actions (cf. Anderson, Garrod, \& Sanford, 1983; Zwaan, Madden, \& Whitten, 2000), rather than to all concepts prior to them. More critically, we can conclude that the reduction in accessibility of characters' former locations in the time and location shift condition resulted from the change in location rather than the change in time.

Error rates. Although the pattern of error rates paralleled the recognition results, they did not differ significantly across conditions (both $F_{\mathrm{s}}<1$ ).

Reading times. Reading times on the last line of each passage, the "postshift" sentence, did not differ signifi-

Table 2

Postshift Sentence Reading Times and Probe Response Times (in Milliseconds) and Error Rates: Experiments 1 and 2

\begin{tabular}{lccc}
\hline \multicolumn{1}{c}{ Condition } & $\begin{array}{c}\text { Reading } \\
\text { Time }\end{array}$ & $\begin{array}{c}\text { Probe } \\
\text { Response Time }\end{array}$ & $\begin{array}{c}\text { Probe } \\
\text { Error Rate }\end{array}$ \\
\hline Experiment 1 & & & \\
$\quad$ No shift & 1,599 & 920 & $14.9 \%$ \\
$\quad$ Time shift & 1,611 & 895 & $12.8 \%$ \\
$\quad$ Time and location shift & 1,572 & 954 & $16.4 \%$ \\
Experiment 2 & & & \\
$\quad$ Time shift & 1,515 & 879 & $8.4 \%$ \\
$\quad$ Time and location shift & 1,482 & 924 & $12.3 \%$ \\
\hline
\end{tabular}


cantly across conditions (both $F \mathrm{~s}<1$ ). Thus, the differences in recognition latencies cannot be attributed to any "spillover" effects from reading the last line.

In summary, the results of Experiment 1 provide support for the online-updating hypothesis. After a shift in location, a protagonist's former location was less accessible in memory than that protagonist's current location. This occurred despite the fact that there was no coherence break that required readers to search memory for the character's location. In combination with de Vega's (1995) finding that current locations were not more accessible than former locations, the present findings support the hypothesis that the inclusion of location information in a situation model is a function of the same factors that influence the accessibility of text concepts more generally, such as the relevance of the information to the narrative.

\section{EXPERIMENT 2}

In Experiment 2, we tested the generality of the findings of Experiment 1. Do readers include location information in their situation models only when a character's location is highly elaborated? Or does this occur more commonly? To answer this question, we modified the passages from Experiment 1 by eliminating the elaborate location descriptions.

\section{Method}

Participants. Fifty SUNY Binghamton undergraduates participated in exchange for course credit. The data from 5 participants who made more than $20 \%$ errors on recognition probes and 1 participant with too many outliers were replaced.

Materials. Twenty of the experimental passages from Experiment 1 were revised (mean length $=6.3$ sentences, range $=4-9$ sentences) so that the majority of the elaboration about the protagonists' locations was removed (see Example 1). Instead of an average of 4.7 sentences describing the locations, ${ }^{3}$ there were only 2.2 . Furthermore, given the lack of influence of a time shift on the availability of location information in Experiment 1, the no-shift condition was dropped. The same filler passages and probes were used as in Experiment 1 .

Design. The experimental passages were randomly assigned with two constraints: (1) Each participant saw half of the passages in each of the two conditions, and (2) each version of the passage was presented to half of the participants.

Procedure. The procedure was identical to that in Experiment 1.

\section{Results and Discussion}

Recognition latencies. Mean recognition times are presented in Table 2. Replicating the key finding of Experiment 1 , recognition times were longer for characters' former locations, in the time and location shift condition (924 msec), than for their current locations, in the time shift condition ( $879 \mathrm{msec})$. This was reliable by participants $\left[t_{1}(43)=2.32, S E M=19.31, p=.03\right]$, but failed to reach significance by items $\left[t_{2}(19)=1.51, S E M=20.44\right.$, $p=.15]$. Despite the elimination of the majority of the location elaboration, the advantage $(45 \mathrm{msec})$ for characters' current locations over their old locations was only slightly less than in Experiment $1(59 \mathrm{msec})$.
Error rates. Error rates were lower in the time shift condition $(8.4 \%)$ than in the time and location shift condition $(12.3 \%)\left[t_{1}(43)=2.64, S E M=0.015, p=.01\right.$; $\left.t_{2}(19)=1.79, S E M=0.022, p=.09\right]$. These results are consistent with the recognition latency results, again suggesting that a character's former location is less accessible in memory after a location shift.

Reading times. Reading times on the last line of the passages, the "postshift" sentence, did not differ significantly (both $p s>.41$ ). Thus, the differences in recognition latencies cannot be attributed to any "spillover" effects from reading the last line.

These results allow us to broaden our conclusions. In the present set of passages, location information was not highly elaborated nor was it emphasized through the use of a specialized task or through instructions. However, even under these conditions, support was found for the online-updating hypothesis. It is not the case that support for the online-updating hypothesis can be found only when location information is highly elaborated. Although the influence of a number of additional text factors, such as the importance of the narrative location to characters' goals, needs to be investigated, the present results suggest that readers update location information in their situation models in a variety of circumstances.

\section{EXPERIMENT 3}

The results of Experiment 1 demonstrate that when location information is highly salient, following a location shift a protagonist's current location is more accessible in memory than the protagonist's former location. In Experiment 2 , we extended this finding by replicating the pattern of results in passages in which the elaboration of location information was substantially reduced. Finally, in Experiment 3, we sought to extend the finding in one further way. In both Experiments 1 and 2, the reduction in accessibility of former locations was found only one sentence after a location shift. Thus, we asked whether the advantage for a protagonist's current location was only short lived, or whether it would be maintained in a situation model even when it had not been referred to recently.

To answer this question, we examined reading times on sentences that each contained a definite noun phrase that referred to an entity that was highly typical of the protagonist's current or former location. For example, after describing a character as being in a forest, the target sentence was "The trees provided nice shade." The ease with which the target sentence is processed should reflect the accessibility of the location information in memory. Walker and Yekovich (1987) found, for example, that script-appropriate objects (e.g., candles in a birthday party narrative) could be referred to with a definite noun phrase and processed with equal ease regardless of whether they had been introduced explicitly.

Passages appeared in three conditions (see Table 3). In all three versions, the characters were described as being in an "original" location (e.g., a forest), and later in the passage a target sentence included a definite reference 
Table 3

Sample Passage From Experiment 3

Introduction and Placement

Maria and Bill went for a day hike in a state forest about an hour away from their home. It was a really hot and sunny day, but they hoped it would still be fun.

Stay-Focused Condition Continuation

After a several hour hike, they say down to eat lunch.

\section{Stay-Backgrounded Condition Continuation}

After a several hour hike, they sat down to eat lunch. It was terrible how out of shape they were, and they were both still in their 20s. Bill swore he would get back to playing hockey and Maria said she'd start running.

\section{Go-Backgrounded Condition Continuation}

After a several hour hike, they went home and collapsed. It was terrible how out of shape they were, and they were both still in their 20s. Bill swore he would get back to playing hockey and Maria said she'd start running.

\section{Pretarget Line}

They both felt exhausted.

\section{Target Line}

The trees provided nice shade.

\section{Conclusion}

Stay conditions: And this was a nice break from their hike.

Go condition: So neither of them had gotten a sunburn.

to an entity typically found in that location (e.g., "The trees provided nice shade."). Two factors were manipulated. First, characters either stayed in the original location (stay) or moved to a new location ( $g o$ ). Second, the target sentence either appeared when the original location was still in focus (focused) or after several lines of backgrounding that changed the focus (backgrounded).

The critical condition was the stay-backgrounded condition, in which the characters remained in their original location, but several lines of backgrounding intervened between the description of their location and the target sentence. These lines shifted the focus of the narrative away from the action occurring in the original location to some aspect of the characters' thoughts or traits (e.g., "It was terrible how out of shape they were ..."). If location information is kept accessible only briefly after it is described, this backgrounding material, which neither explicitly nor implicitly referred to location, should cause the location to be dropped from the situation model. Given this, when readers encounter the target sentence they should have difficulty processing a definite reference to an entity that is not part of the current focus of the narrative. On the other hand, if characters' current location remains in the situation model even when it has not been mentioned for several sentences, the processing of the target entity should proceed easily.

There were two control conditions. The first was a stay-focused condition. Unlike in the stay-backgrounded condition, the target sentence appeared shortly after the characters' location was described. Although a couple of lines of text intervened between the last mention of the location and the target sentence, the location was salient in the intervening material ("It was a really hot and sunny day ..."). Thus, the location information should be easily accessible when the noun phrase is encoun- tered, making processing easy (e.g., Walker \& Yekovich, 1987). If location information is maintained in the situation model in the stay-backgrounded condition, despite the shift in focus, reading times on the target line should not differ from those in the stay-focused condition.

The second control condition was the go-backgrounded condition. In this condition, characters were no longer in the original location but had moved to a new location (“. . . they went home and collapsed."). In addition, the same backgrounding as in the stay-backgrounded condition intervened between the location information and the target sentence. According to the online-updating hypothesis, and given the results of Experiments 1 and 2, the change in the characters' location should cause their former location to be reduced in accessibility. This should result in a slowdown on the target sentence due to the difficulty in establishing coreference between the noun phrase and a referent that is not present in the narrative setting (e.g., Anderson et al., 1983; Rinck \& Bower, 1995). Moreover, for narratives like the ones used in this experiment, the use of the simple past tense is appropriate only for entities that were present in the narrative setting (i.e., the "narrative now"; Almeida, 1995).

\section{Method}

Participants. Thirty-two SUNY Binghamton undergraduates participated in exchange for course credit or $\$ 5$. One participant made more than $20 \%$ errors on comprehension questions, and 1 participant did not follow instructions. The data from these 2 participants were replaced. Therefore, analyses were based on 30 participants.

Materials. Eighteen experimental passages were used, with three versions of each (see Table 3). All three versions began with the introduction of the characters, the establishment of the narrative location (e.g., a forest), and a description of some action (e.g., a hike) occurring in that location. In the stay-backgrounded condi- 
tion, the introductory episode was concluded with an action occurring in the narrative location (e.g., ". . they sat down to eat lunch.”), followed by two or three sentences of backgrounding material that were neutral with respect to the location, but that served to shift the focus of the passage. In the stay-focused condition, the introductory episode was concluded with the same action, but there was no backgrounding. In the go-backgrounded condition, the introductory episode was concluded with an action that changed the narrative location (e.g., ". . . they went home ...") and the same backgrounding as in the stay-backgrounded condition. All three versions then continued with the same pretarget sentence that was neutral with respect to location and the same target sentence that referred to an entity typical of the original location. Finally, the last line provided a sensible completion, which sometimes differed across conditions. The wording used to describe the passage of time was equated across all three conditions.

In addition to the experimental passages, there were 21 filler passages, a subset of those used in Experiment 1. To encourage careful reading, after 10 of the filler passages a two-alternative forced choice comprehension question was presented.

Design. The design was the same as in Experiment 1.

Procedure. The procedure was the same as in Experiment 1, with the following exceptions: (1) No recognition probe task was used; (2) instead of having to write continuations, at five points during the experiment participants were instructed to recall "what happened" in one of the previous 7 passages, given the first line of the passage as a recall cue; and (3) only 10 out of 39 passages were followed by comprehension questions.

\section{Results and Discussion}

For both the pretarget and target lines, outlying reading times were removed for each item using Tukey's (1977) procedure. This resulted in the exclusion of $8.1 \%$ and $6.9 \%$ of the pretarget and target reading times, respectively.

Pretarget sentence. Reading times on the pretarget sentence were $1,768,1,780$, and $1,765 \mathrm{msec}$ in the staybackgrounded, stay-focused, and go-backgrounded conditions, respectively. These times did not differ reliably (both $F_{\mathrm{S}}<1$ ). Thus, any differences in the reading times on the target sentence were not due to extraneous factors such as passage length or differences in the difficulty of processing the pretarget sentence.

Target line. With respect to the main comparison of interest, the participants read the target sentence as quickly in the stay-backgrounded condition as in the stay-focused condition $(1,461 \mathrm{vs.} 1,449 \mathrm{msec}$; both $p$ s $>$ .7). Thus, a character's location remained as accessible in the stay-backgrounded condition despite the change in focus and additional backgrounding. Readers had no more difficulty processing the target concept (e.g., the trees) when the protagonist's location was out of focus than when it was in focus. We can conclude that, at least when location is salient in a narrative, it is updated immediately and remains accessible for several sentences downstream, being maintained in the situation model.

In contrast, it was more difficult to process the target sentence when it referred to an object found in a character's prior location than when it was found in the character's current location; reading times on the target sentence were $111 \mathrm{msec}$ longer in the go-backgrounded condition $(1,572 \mathrm{msec})$ than in the stay-backgrounded condition $(1,461 \mathrm{msec})\left[t_{1}(29)=2.33, S E M=47.73 ; t_{2}(17)=1.96\right.$,
$S E M=55.25, p=.067]$. This extends the results of Experiments 1 and 2, where, shortly after a change in location, characters' former locations were less accessible than their current locations. In the present experiment, updating effects were found even several sentences after a location shift.

\section{GENERAL DISCUSSION}

In three experiments, we examined the processes involved in encoding location information in narratives and the nature of the resulting memory representation. Despite de Vega's (1995) conclusion that the updating of location information occurs only to repair a coherence break, we hypothesized that readers should be flexible with respect to the information that they include in their situation models. Given this, the probability of including location information in readers' situation models should be a function of the same factors that influence the accessibility of text concepts more generally, such as the amount of elaboration and the centrality of that information to the narrative.

Consistent with this hypothesis, in Experiment 1, when location information was highly elaborated and integrated into the action of the passages, recognition latencies were significantly longer for characters' former locations than for their current locations. In Experiment 2, this result was replicated even though the elaboration of location information was reduced. These results provide support for the online-updating hypothesis (de Vega, 1995); the representation of a character's old location was immediately suppressed, or deactivated, when that character moved to a new location. Thus, readers carefully tracked location even when there were no special task demands and when access to this information was not needed for maintaining the coherence of a text.

In addition to investigating the processes involved in the updating of location information, in Experiment 3 we found that location information retains some sort of privileged status in memory, remaining easily accessible even several sentences after its last explicit mention. When participants encountered a definite reference to an entity typically found in the character's location, it was as easy to process when the last mention of the location had been backgrounded by several sentences of text that changed the focus as when there was very little backgrounding.

The present set of findings can be viewed with respect to the more general debate over what types of information are represented in a reader's situation model (Zwaan \& Radvansky, 1998). It appears that readers are flexible and efficient in their assessment of the relevance of information to a narrative. For instance, location information may or may not be included in a situation model, depending on factors such as the salience of location and readers' comprehension strategies and skills. With materials in which location information was neither elaborately described nor central to the text, de Vega (1995; see also Hakala, 1999) found that the updating of spatial 
information was optional, being done only to maintain text coherence. In contrast, in the present experiments, when location information was relevant to the characters' goals and actions, readers immediately updated their situation model. In a similar manner, it has been found that after a time shift, information that is no longer relevant to a narrative is suppressed (Anderson et al., 1983; Carreiras et al., 1997; Magliano \& Schleich, 2000; Zwaan, 1996), whereas information that continues to be relevant remains active (Experiment 1; Anderson et al., 1983; Magliano \& Schleich, 2000; Zwaan et al., 2000).

Clearly, these experiments represent a first step. The influence of a number of text factors on the salience of location information remains to be investigated. The materials used in the present experiments differed in a number of ways from de Vega's (1995), making it unclear exactly what text factors are necessary, or sufficient, for readers to maintain location information in their situation models. For example, the present materials contained greater elaboration of location information and integrated that information with the characters' goals. Although the results of Experiment 2 suggest that the elaboration alone was not critical, the integration of the location with the characters' goals may have been. Current theoretical accounts of situation model construction (e.g., Gernsbacher, 1990; Zwaan, Magliano, \& Graesser, 1995) suggest that the co-occurrence of a location shift with a character's attempt to satisfy a goal may provide a strong cue to the reader that the situation model needs to be updated. This theoretical framework also suggests that the co-occurrence of location shifts with time shifts, as in the time and location shift conditions in Experiments 1 and 2, may be important (Magliano, Miller, \& Zwaan, in press), with time shifts acting as an additional cue that the situation model needs to be updated. Another difference between the current set of materials and de Vega's was the relative size of the location shifts (cf. Rinck \& Bower, 1995). Although this was not systematically manipulated, in many cases the location shifts in the present materials were between nonadjacent locations, whereas in de Vega's the location shifts were always between adjacent locations. It is possible that the greater the size of a location shift, the greater the probability that readers will update their situation model. Finally, in addition to the differences in the materials, there were a number of methodological differences between the present experiments and de Vega's. Perhaps most importantly, in the present experiments, reading was self-paced, whereas in de Vega's, reading was experimenter paced. In addition to contributing to very different reaction times in the recognition task (870-950 msec for our participants, 1,500$1,800 \mathrm{msec}$ for de Vega's), this may have somehow altered the nature of the reading experience.

More generally, although the present findings demonstrate that there are conditions under which readers maintain location information in their situation models, for a complete theory of discourse processing an understanding is needed of the influence of the full range of text and reader characteristics. For example, although we found that location information remained salient several sentences after its last mention, with sufficient backgrounding between the description of a character's location and a reference to a location-typical entity, it is possible that the character's location would no longer remain easily accessible. Second, not only might the amount of backgrounding influence the accessibility of location information, but the nature of that backgrounding might also play a role; a complete change in focus might lead to a greater reduction in accessibility. Third, the uniqueness or distinctiveness of a location (e.g., a haunted house vs. an office) might also influence its longevity in a situation model. Fourth, the generalizability of the present results to narratives having more than two locations is an open question. Fifth, the amount of spatial detail that is included in a narrative may influence the probability of encoding; readers might encode spatial information carefully only if it was easy to do. Consistent with this, Zwaan and van Oostendorp (1993) found that unless instructed to do so, readers did not carefully track spatial information in a mystery novel that described a crime scene with many spatial details, and Mani and JohnsonLaird (1982) found that readers did not construct a spatial representation when there was more than one way to do so. Finally, there might be differences between readers, with people who were more invested in a story, or who were more proficient readers, being more likely to maintain location information in their situation models.

By what process might readers manage the accessibility of spatial information in their situation models? According to a variety of theories of situation model construction (Garrod \& Sanford, 1990; Gernsbacher, 1990; Zwaan \& Radvansky, 1998), readers maintain tokens in working memory representing the important entities in a discourse, such as the protagonists and some of their essential characteristics. These entities are considered to be foregrounded or in explicit focus (Garrod \& Sanford, 1990; Zwaan \& Radvansky, 1998). In addition to the tokens in explicit focus, important background information about the discourse such as time, location, and goals (Zwaan \& Radvansky, 1998), is held in long-term working memory (Ericsson \& Kintsch, 1995; Zwaan \& Radvansky, 1998; see Gernsbacher, 1990, for a similar proposal) or implicit focus (Garrod \& Sanford, 1990), being kept easily accessible through the maintenance of pointers from the tokens in working memory. Given the discrepancy between the present findings and de Vega's (1995), it appears that location information might or might not be included in implicit focus depending on its importance to the narrative. If location information is included in implicit focus, when a location shift occurs the pointer from the character to the old location is deactivated or suppressed (Gernsbacher, 1990), resulting in the removal of the old location from implicit focus and a reduction in its accessibility. At the same time, the new location enters into implicit focus, with a pointer being created from the 
character's token in explicit focus. In addition, as long as the focused character remains in a particular setting, their location should remain easily accessible.

In summary, the present findings add to a growing body of results that suggest that readers are able to keep track of a variety of situational indices while reading. Time shifts, both explicit (Anderson et al., 1983; Zwaan, 1996) and in the form of verb aspect and tense (Carreiras et al., 1997; Magliano \& Schleich, 2000), have been shown to lead readers to deactivate or suppress no longer pertinent information. The same has been shown to be true of episode shifts (Gernsbacher, 1990). Although the present research leaves open the question of how much spatial detail readers routinely encode (Zwaan \& van Oostendorp, 1993), in combination with converging evidence from Black et al. (1979) and O'Brien and Albrecht (1992), it suggests that readers are able to encode at least gross spatial detail under naturalistic conditions, using location shifts as cues to update their representations. Additional research is needed in order to determine the range of conditions under which location information is encoded into readers' situation models and the influential text variables.

\section{REFERENCES}

Almeida, M. J. (1995). Time in narratives. In J. F. Duchan, G. A Bruder, \& L. E. Hewitt (Eds.), Deixis in narrative: A cognitive science perspective (pp. 159-189). Hillsdale, NJ: Erlbaum.

Anderson, A., Garrod, S. C., \& SAnford, A. J. (1983). The accessibility of pronominal antecedents as a function of episode shifts in narrative text. Quarterly Journal of Experimental Psychology, 35A, 427-440.

Black, J. B., Turner, T. J., \& Bower, G. H. (1979). Point of view in narrative comprehension, memory, and production. Journal of Verbal Learning \& Verbal Behavior, 18, 187-198.

Bransford, J. D., Barclay, J. R., \& Franks, J. J. (1972). Sentence memory: A constructive versus interpretive approach. Cognitive Psychology, 3, 193-209.

Carreiras, M., Carriedo, N., Alonso, M. A., \& Fernández, A. (1997). The role of verb tense and verb aspect in the foregrounding of information during reading. Memory \& Cognition, 25, 438-446.

DE VEGA, M. (1995). Backward updating of mental models during continuous reading of narratives. Journal of Experimental Psychology: Learning, Memory, \& Cognition, 21, 373-385.

ERICSSON, K. A., \& KinTSCH, W. (1995). Long-term working memory. Psychological Review, 102, 211-245.

GARRod, S., \& SANFORD, A. J. (1982). The mental representation of discourse in a focussed memory system: Implications for the interpretation of anaphoric noun phrases. Journal of Semantics, 1, 21-41.

GARROD, S., \& SANFORD, A. J. (1990). Referential processes in reading: Focusing on roles and individuals. In D. A. Balota, G. B. Flores d'Arcais, \& K. Rayner (Eds.), Comprehension processes in reading (pp. 465-485). Hillsdale, NJ: Erlbaum.

GERNSBACHER, M. A. (1990). Language comprehension as structure building. Hillsdale, NJ: Erlbaum.

Glenberg, A. M., Meyer, M., \& Lindem, K. (1987). Mental models contribute to foregrounding during text comprehension. Journal of Memory \& Language, 26, 69-83.

Haenggi, D., Kintsch, W., \& Gernsbacher, M. A. (1995). Spatial situation models and text comprehension. Discourse Processes, 19, 173-199.

HaKala, C. (1999). Accessibility of spatial information in a situation model. Discourse Processes, 27, 261-280.
JOHNSON-LAIRD, P. N. (1983). Mental models: Toward a cognitive science of language, inference, and consciousness. Cambridge, MA: Harvard University Press.

KInTSCH, W. (1988). The role of knowledge in discourse comprehension: A construction-integration model. Psychological Review, 95, 163-182.

Magliano, J. P., Miller, J., \& ZwaAn, R. A. (in press). Indexing space and time in film understanding. Applied Cognitive Psychology.

Magliano, J. P., \& Schleich, M. C. (2000). Verb aspect and situation models. Discourse Processes, 29, 83-112.

MANi, K., \& Johnson-LaIRD, P. N. (1982). The mental representation of spatial descriptions. Memory \& Cognition, 10, 181-187.

Morrow, D. G., Greenspan, S. L., \& Bower, G. H. (1987). Accessibility and situation models in narrative comprehension. Journal of Memory \& Language, 26, 165-187.

O'Brien, E. J., \& Albrecht, J. E. (1992). Comprehension strategies in the development of a mental model. Journal of Experimental Psychology: Learning, Memory, \& Cognition, 18, 777-784.

Rinck, M., \& Bower, G. H. (1995). Anaphora resolution and the focus of attention in situation models. Journal of Memory \& Language, 34, 110-131.

Schank, R., \& Abelson, R. (1977). Scripts, plans, goals and understanding: An inquiry into human knowledge structures. Hillsdale, $\mathrm{NJ}$ : Erlbaum.

TukeY, J. W. (1977). Exploratory data analysis. Reading, MA: AddisonWesley.

VAn DiJK, T. A., \& KinTsch, W. (1983). Strategies of discourse comprehension. New York: Academic Press.

WALKer,F., \& YeKovich, C. (1987). Activation and use of script-based antecedents in anaphoric reference. Journal of Memory \& Language, 26, 673-691.

ZWAAN, R. A. (1996). Processing narrative time shifts. Journal of Experimental Psychology: Learning, Memory, \& Cognition, 22, 11961207.

Zwaan, R. A., Madden, C. J., \& Whitten, S. N. (2000). The presence of an event in the narrated situation affects its availability to the comprehender. Memory \& Cognition, 28, 1022-1028.

Zwaan, R. A., Magliano, J. P., \& Graesser, A. C. (1995). Dimensions of situation model construction in narrative comprehension. Journal of Experimental Psychology: Learning, Memory, \& Cognition, 21, 386-397.

ZwaAn, R. A., \& Radvansky, G. A. (1998). Situation models in language comprehension and memory. Psychological Bulletin, 123, 162-185.

Zwaan, R. A., Radvansky, G. A., Hilliard, A. E., \& Curiel, J. M. (1998). Constructing multidimensional situation models during reading. Scientific Studies of Reading, 2, 199-220.

ZWAan, R. A., \& VAN OOSTENDORP, H. (1993). Do readers construct spatial representations during naturalistic story comprehension? Discourse Processes, 16, 125-143.

\section{NOTES}

1. These participants had fewer than three observations in one or both conditions after errors and outliers were removed from their data.

2. According to Tukey's (1977) criterion, a score, $X$, is defined as an outlier if $\mathrm{X}>H_{\mathrm{U}}+(1.5) \times\left(H_{\mathrm{U}}-H_{\mathrm{L}}\right)$ or $\mathrm{X}<H_{\mathrm{U}}-(1.5) \times\left(H_{\mathrm{U}}-\right.$ $\left.H_{\mathrm{L}}\right) . H_{\mathrm{U}}$ and $H_{\mathrm{L}}$ refer to the scores that cut off the upper and lower $25 \%$ of the ranked scores.

3. A sentence was counted as "describing" a location if it made a direct or anaphoric reference to a location, or if it described some aspect of the location.

(Manuscript received August 3, 1999; revision accepted for publication August 7, 2000.) 